\title{
THE SPECTRUM OF SN 1987A IN THE LMC IN JULY 1987
}

\author{
C.C. HUANG \\ Purple Mountain Observatory \\ Academia Sinica \\ 210008 Nanjing, China
}

\begin{abstract}
Spectroscopic observations of SN 1987A in the LMC in July 1987 are presented. The appearance of SN1987A's optical spectrum was dominated by strong $\mathrm{H} I$ and $\mathrm{Na} I$ lines showing broad PCygni profiles. The nebular emission lines of [O I] 6300, 64 appeared in mid-July. The enhancement of sprocessed elements of $\mathrm{Ba}$ II and $\mathrm{Sr}$ II in SN 1987A is briefly discussed.
\end{abstract}

Since the explosion of SN 1987A in 1987 February 23, spectroscopic observations of it have been reported. Many described its early spectral evolution (e.g. Phillips 1988, Hearnshaw et al. 1988). We present spectroscopic observations of SN 1987A in July 1987 when it began its transition to the nebular phase. We obtained about 30 red and blue spectra from July 27 to 29 using a grating spectrograph attached to the $0.61-\mathrm{m}$ Boller and Chivens telescope of Mt John University Observatory. Hearnshaw et al. (1988) describe in detail the observing instrument and techniques. The plates were measured with the PDS densitometer at Purple Mountain Observatory and data reduction carried out using the Tololo-Vienna interaction image processing system.

Figs 1 and 2 give density plots for selected red and blue spectra. In July the appearance of SN1987A's spectrum was dominated by strong $\mathrm{H}$ I and Na I lines showing broad P-Cygni profiles. Although there were no great changes in the spectrum in July 1987, during our observations the emission feature around $\lambda 6300$ showed dramatic variability in configuration and strength. The appearance of this emission was quite different between 9-18 July; its tooth structure of July 9, which had been present for some time, disappeared on July 18 . The emission then enhanced, and changed to a double-peaked emission line due to [O I] 6300,64; then these nebular lines rapidly increased in intensity and SN1987A began to evolve into its nebular phase (Couch 1988).

Over much of the first seven months since SN1987A's core collapse, the optical and infrared spectra were characterized by strong absorption lines of s-process elements $\mathrm{Ba}$ II and $\mathrm{Sr}$ II (Phillips 1988). Our optical observations are consistent with his results. Besides the two lines Ba II $\lambda \lambda 4554,6142$ indicated by Phillips in his optical spectra, we measured another line at $\lambda 4934$ due to $\mathrm{Ba}$ II $4934+\mathrm{Fe}$ II 4924 (Williams 1987). The radial velocity of the absorption component of the line is $-1819 \mathrm{~km} \mathrm{~s}^{-1}$ which agrees with that of $\mathrm{Ba}$ II $\lambda 6142$. The radial velocities of the absorption components of Ba II $\lambda \lambda 4554,6142$ are $-2245 \mathrm{~km} \mathrm{~s}^{-1}$ and-1889 $\mathrm{km} \mathrm{s}^{-1}$ resp. The higher radial velocity of $\mathrm{Ba}$ II $\lambda 4554$ is probably affected by the severe line blanketing which depresses the continuum just blueward of line centre (cf. Phillips 1988). The radial velocity of the absorption component of the other s-process element $\mathrm{Sr}$ II $\lambda 4215$ is about $-2584 \mathrm{~km} \mathrm{~s}^{-1}$. As Williams (1987) points out, the presence of the $\mathrm{Ba}$ II and $\mathrm{Sr}$ II lines requires s-process element enhancements in the progenitor of SN1987A, very important in determining the former's evolutionary status. 


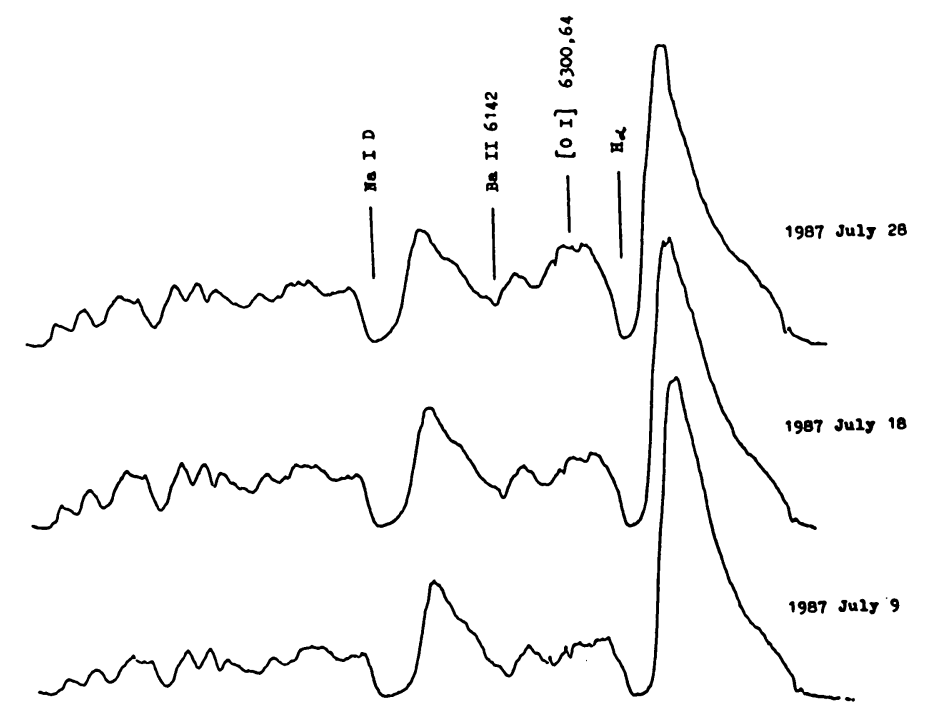

Figure 1. Density plots of the red spectra of SN 1987A

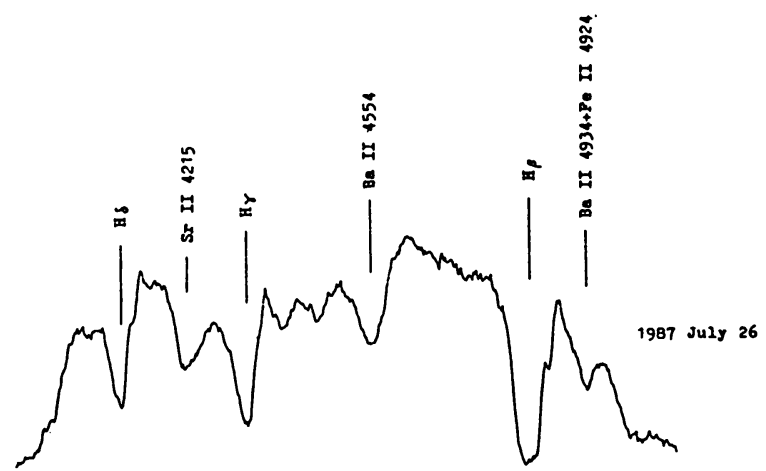

Figure 2. Density plots of the blue spectrum of SN 1987A

\section{Acknowledgments}

I thank Dr. J.B. Hearnshaw very much for precious observing time and Mr. Alan Gilmore for valuable help. This research was supported in part by the National Foundation of Natural Science.

\section{References}

Couch, W.J. (1988), Supernova 1987A in the Large Magellanic Cloud, M. Kafatos and A.G. Michalitsianos (eds.) (Cambridge University: Cambridge) p.18.

Hearnshaw, J.B., McIntyre, V.J., Gilmore, A.C. and Haar, J. (1988), Vistas in Astron. 31, 243. Phillips, M.M. (1988), Supernova 1987A in the Large Magellanic Cloud,. M. Kafatos and A.G. Michalitsianos (eds.) (Cambridge University: Cambridge) p.16.

Williams, R.E. (1987), Astrophys. J. 320, L117. 